Session 3232

\title{
Meeting the ABET Computer Competency Requirement in the Department of Electrical and Computer Engineering at Tennessee State University
}

\author{
M. Bodruzzaman, S. S. Devgan, and S. Zein-Sabatto \\ Tennessee State University
}

\begin{abstract}
Introduction
In the Information Age, access to timely information in appropriate form is power. Engineers of tomorrow must be proficient in developing computer tools and applications to facilitate access to information anywhere any time. ABET criteria for computer use and computer facilities in engineering curriculum and specifically Electrical, Electronic(s), Computer and similarly named engineering programs provides the minimum requirements [1]. The Engineering Criteria 2000 lists similar requirements in the form of desired competencies for an engineering graduate [2]. Recent ABET visit and our desire to review, evaluate and plan for development of computer facilities within the College of Engineering and Technology and the Department of Electrical and Computer Engineering in specific, provided us an opportunity to access our current level of computer use and computer facilities. This evaluation is listed in tabular form. This also provided us an opportunity to develop guidelines for meeting ABET computer use and facilities requirements and plan for future developments in our program to meet Engineering Criteria 2000 requirements. The Electrical Engineering curriculum, and its concentration in Computers, requires at least two student written computer projects for all o its major courses. Wordprocessed technical reports and oral presentation have now became a norm in our program. At least two laboratories are equipped with latest model computers with major higher level programming language compilers, and application software such as Matlab, LabVIEW, LabWindow and other simulation and modeling software. Students have access to Internet via Netscape browser for information retrieval and use of resources that are available elsewhere. Computer-based data acquisition experience is acquired in the Control Systems, Circuits, and Electrical Systems Design Laboratories. Additionally many students work with faculty on various federal and industry funded research projects and use computers for real-time data acquisition, signal/image processing, energy related research, predictive maintenance, intelligent controls and robotics areas. This paper examines the computer competency skills acquired by our students towards meeting ABET criteria and presents a model plan for evaluating such competencies.
\end{abstract}

\section{The Required Level of Competency}

After reviewing the ABET Criteria for accrediting programs in Engineering in general and Electrical, Electronic(s), Computer and similarly named engineering programs in specific, we identified that the following topics be considered in evaluating the desired competencies that an electrical and/or computer engineering graduate should have acquired as part of the undergraduate curriculum requirements. These are listed under ABET criteria IV.C.3.g and IV.C.6.c.

- Experience with advanced features of at least one major operating system: This requirement could be satisfied if the student had experience with Windows 95, Windows NT 
or Unix operating system including networking as a part of computer programming and/or a software engineering course.

- Proficiency in use of modern scientific tools including computers: Document preparation and report writing using one or more scientific word processing tools such as: MS-Word'97, Word Perfect, Latex etc. should be a standard requirement for all engineering students. Proficiency in document transfer using e-mail attachment, FTP etc. should also be integrated into the curriculum. Student must be required to use computer-generated overheads or presentations using modern tools such as Power Point, Harvard Graphics as part of oral communication skills requirement.

- Programming Languages: Experience and skills in writing and debugging programs in several of the modern programming languages such as FORTRAN-77, Fortran-90, C, C++, Pascal, QBasic, Visual Basic, Ada, Java, etc. for technical calculations, information transfer and engineering problem solving should be integrated throughout the curriculum. Program should assure that student developed computer programs follow the formal software development and design requirements.

- Modeling, Simulation and Analysis: Experience in the use of modeling, simulation and computer-assisted design software packages such as Matlab Toolboxes, Mathematica, Finite Element Analysis (FEA), PSpice, LabVIEW, EMTP/ATP, EasyPower, CFD, etc. in the design and analysis of a physical engineering system should be part of curriculum requirements.

- Data Acquisition, Processing and Analysis: Use of computer-assisted data acquisition, processing and analysis, using any of the data analysis software packages such as Statistical Package (SAS), spread sheet (Lotus, Excel etc.), Matlab Toolbox, LabVIEW, etc. should be integrated into various laboratory and student design project requirements.

- Computer Facilities and Support: Well-maintained modern computer facilities should be accessible to all students well beyond the normal working hours to meet student needs. Welltrained staff and laboratory assistants should be available to supplement university computer center staff.

\section{Review of Existing Computer Competencies and Facilities}

An analysis on our Electrical Engineering curriculum with respect to meeting ABET program criteria was carried out. The process involved review of ABET course outlines, detailed course outlines and review of computer assignments for all required and technical elective courses. Table 1 lists the coverage of computer use in engineering courses while Table 2 lists the similar coverage in required and technical elective courses under the B.S.E.E. degree and the concentration in computers under this degree program. Those courses that meet the various components of above criteria are identified with an " $\mathrm{X}$ " mark. This does not, however, indicate the level of competence achieved but that there is evidence of coverage.

The existing hardware/software facilities in the College of Engineering and Technology and the Department of Electrical and Computer Engineering are given in Table 3. The criteria used in Table 3 was developed from the ABET requirements, Computer Literacy Guide of the College Board publication, and our College Computer Committee criteria. Our current support staff includes one technician for maintaining the College facilities and one technician for electrical engineering facilities. In addition we have undergraduate and graduate teaching assistants who keep the labs open for long hours. They supplement the University computer staff and facilities. 


\section{New Initiatives and Future Plans}

We have initiated a concentration in Computers under the B.S.E.E. program and a M.S. degree in Computer and Information Systems Engineering. These programs use systems approach to development of integrated computer hardware and software systems. We have submitted a proposal to establish a Unix-based software development laboratory and plan to seek additional simulation and analysis software packages that will be integrated into our curriculum.

An analysis of the three tables shows only the existence of the computer usage under each criterion and the existence of the hardware/software facilities in the department. The level of competency or skills were not measured and/or evaluated in this report. We plan to carry out a detailed course content evaluation and survey other campus-based computer training programs, seminars, and tutorials available to our students, to determine the level of competency achieved in the area of computer use.

\section{Conclusions}

An analysis of our program with respect to computer use has been very beneficial and will be carried out further to document the level of competency achieved by every student. This kind of documentation and analysis will be needed to satisfy Engineering Criteria 2000 as it relates to criteria $3 \mathrm{k}$.

\section{References}

1. "Criteria For Accrediting Programs in Engineering in the United States (1996-97)," Engineering Accreditation Commission, ABET, Inc. Baltimore, MD.

2. Engineering Criteria 2000" $2^{\text {nd }}$ edition. Engineering Accreditation Commission of the Accreditation Board for Engineering and Technology.

3. "Academic Preparation For College," The College Board., Mount Vernon, IL.

4. Course outlines (1996-97), for all courses of AE, CE, EE and ME, College of Engineering and Technology, Tennessee State University, Nashville, TN.

5. Course Descriptions (1996-97), prepared for ABET for all courses offered by AE, CE, EE and ME departments of the College of Engineering and Technology, Tennessee State University, Nashville, TN. Fall 1997.

\section{Biographical Information}

Dr. MOHAMMAD BODRUZZAMAN is an Associate Professor of Electrical and Computer Engineering. His areas of research interest are Pattern Recognition, Signal Analysis, Wavelet Applications to signal analysis and health monitoring. He has published over seventy refereed and non-refereed papers and a member of IEEE and honor societies.

Dr. S. S. DEVGAN is Professor and Head of Electrical and Computer Engineering. His areas of research interest are electrical power system analysis, distribution system design, hybrid wind electric systems design, computer networks and systems engineering. He is a senior member of IEEE and is a Registered Professional Engineer.

Dr. M.S. ZEIN-SABATTO is an Associate Professor of Electrical and Computer Engineering. His areas of interest include Intelligent Control Systems,. Robotics and Predictive Maintenance. He is active in funded research and has published over 36 refereed and non-refereed conference papers. He is serving as Vice-President of IEEE Nashville Section. 
Table 1

COMPUTER COMPETENCY IN GENERAL ENGR COURSES

\begin{tabular}{|c|c|c|c|c|c|c|c|c|}
\hline & \multicolumn{8}{|c|}{ CRITERIA FOR COMPUTER COMPETENCY } \\
\hline $\begin{array}{c}\text { Course Number } \\
\text { and } \\
\text { Title }\end{array}$ & $\begin{array}{l}\text { MS-Dos, } \\
\text { Window- } \\
95 \text { or } \\
\text { UNIX Op. } \\
\text { Sys. }\end{array}$ & $\begin{array}{l}\text { Report } \\
\text { Writing } \\
\text { Using } \\
\text { Computer }\end{array}$ & $\begin{array}{l}\text { Technical } \\
\text { Presenta- } \\
\text { tion } \\
\text { Using } \\
\text { Computer }\end{array}$ & $\begin{array}{l}\text { Computer } \\
\text { Graphics }\end{array}$ & $\begin{array}{l}\text { Structured } \\
\text { Program, (C, } \\
\text { C++, F-77, } \\
\text { Pascal, } \\
\text { Qbasic etc.) }\end{array}$ & $\begin{array}{l}\text { Design, } \\
\text { Modeling, } \\
\text { Simulation, } \\
\text { Analysis, } \\
\text { Using } \\
\text { Package Tools }\end{array}$ & $\begin{array}{l}\text { Design, } \\
\text { Modeling, } \\
\text { Simulation, \& } \\
\text { Analysis, by } \\
\text { Writing Own } \\
\text { Program } \\
\end{array}$ & $\begin{array}{l}\text { Data } \\
\text { Acquisition } \\
\text { Analysis } \\
\text { Using Tool or } \\
\text { Writing } \\
\text { Program } \\
\end{array}$ \\
\hline $\begin{array}{l}\text { ENGR 100L (1) } \\
\text { Intro. to Engineering I }\end{array}$ & $\mathrm{X}$ & $\mathrm{X}$ & - & $\mathrm{X}$ & - & - & - & - \\
\hline $\begin{array}{l}\text { ENGR 101L (1) } \\
\text { Intro. to Engineering II }\end{array}$ & $\mathrm{X}$ & $\mathrm{X}$ & $\mathrm{X}$ & $\mathrm{X}$ & - & $\mathrm{X}$ & - & - \\
\hline $\begin{array}{l}\text { ENGR 113L (3) } \\
\text { Engr. Design Graphics }\end{array}$ & $\mathrm{X}$ & $\mathrm{X}$ & $\mathrm{X}$ & $\mathrm{X}$ & - & $\mathrm{X}$ & - & - \\
\hline $\begin{array}{l}\text { ENGR 200 (3) } \\
\text { Circuits I }\end{array}$ & $\mathrm{X}$ & $\mathrm{X}$ & - & $\mathrm{X}$ & $\mathrm{X}$ & - & $\mathrm{X}$ & - \\
\hline $\begin{array}{l}\text { ENGR 200L (1) } \\
\text { Circuits I Lab }\end{array}$ & $X$ & $\mathrm{X}$ & - & $\mathrm{X}$ & - & $\mathrm{X}$ & - & $X$ \\
\hline $\begin{array}{l}\text { ENGR } 201(3) \\
\text { Thermodynamics }\end{array}$ & - & - & - & - & - & - & - & - \\
\hline $\begin{array}{l}\text { ENGR } 211 \text { (4) } \\
\text { Engineering Mechanics I }\end{array}$ & - & - & - & - & - & - & - & - \\
\hline $\begin{array}{l}\text { ENGR } 212(4) \\
\text { Mechanics I }\end{array}$ & - & - & - & - & - & - & - & - \\
\hline $\begin{array}{l}\text { ENGR } 213 \text { (4) } \\
\text { Engineering Mechanics II }\end{array}$ & - & - & - & - & - & - & - & - \\
\hline $\begin{array}{l}\text { ENGR } 221(3) \\
\text { Engr. Fortran Prog. }\end{array}$ & $X$ & $\mathrm{X}$ & - & - & $X$ & - & $X$ & - \\
\hline $\begin{array}{l}\text { ENGR } 223(3) \\
\text { Engineering C Prog. }\end{array}$ & $X$ & $\mathrm{X}$ & $\mathrm{X}$ & - & $\mathrm{X}$ & - & $\mathrm{X}$ & - \\
\hline $\begin{array}{l}\text { ENGR } 225 \text { (4) } \\
\text { Engr. Transport Phenom. }\end{array}$ & $X$ & $\mathrm{X}$ & - & - & - & - & $X$ & - \\
\hline $\begin{array}{l}\text { ENGR } 320(3) \\
\text { Intro. to Engr. Design }\end{array}$ & $X$ & $\mathrm{X}$ & $\mathrm{X}$ & - & $\mathrm{X}$ & - & $X$ & - \\
\hline $\begin{array}{l}\text { ENGR } 330(3) \\
\text { Material Science }\end{array}$ & - & - & - & - & - & - & - & - \\
\hline $\begin{array}{l}\text { ENGR } 340 \text { (3) } \\
\text { Numerical Analysis }\end{array}$ & $X$ & $\mathrm{X}$ & - & - & $\mathrm{X}$ & - & $\mathrm{X}$ & - \\
\hline
\end{tabular}




\section{Table 2}

\section{COMPUTER COMPETENCY IN EE COURSES}

\begin{tabular}{|c|c|c|c|c|c|c|c|c|}
\hline & \multicolumn{8}{|c|}{ CRITERIA OF COMPUTER COMPETENCY/SKILLS MEASURE } \\
\hline $\begin{array}{c}\text { Course Number } \\
\text { and } \\
\text { Title }\end{array}$ & $\begin{array}{l}\text { MS-Dos, } \\
\text { Window- } \\
95 \text { or } \\
\text { UNIX Op. } \\
\text { Sys. }\end{array}$ & $\begin{array}{l}\text { Report } \\
\text { Writing } \\
\text { Using } \\
\text { Computer }\end{array}$ & $\begin{array}{l}\text { Technical } \\
\text { Presenta- } \\
\text { tion } \\
\text { Using } \\
\text { Computer }\end{array}$ & $\begin{array}{l}\text { Computer } \\
\text { Graphics }\end{array}$ & $\begin{array}{l}\text { Structured } \\
\text { Program, } \\
\text { (C, C++, F- } \\
\text { 77, Pascal, } \\
\text { Qbasic etc.) }\end{array}$ & $\begin{array}{l}\text { Design, } \\
\text { Modeling, } \\
\text { Simulation, } \\
\text { Analysis, } \\
\text { Using } \\
\text { Package Tools }\end{array}$ & $\begin{array}{l}\text { Design, } \\
\text { Modeling, } \\
\text { Simulation, \& } \\
\text { Analysis, by } \\
\text { Writing Own } \\
\text { Program }\end{array}$ & $\begin{array}{l}\text { Data } \\
\text { Acquisition } \\
\text { Analysis } \\
\text { Using Tool } \\
\text { or Writing } \\
\text { Program }\end{array}$ \\
\hline $\begin{array}{l}\text { EE } 212 \text { (3) } \\
\text { Circuits II }\end{array}$ & $\mathrm{X}$ & $\mathrm{X}$ & - & - & $\mathrm{X}$ & $\mathrm{X}$ & $\mathrm{X}$ & - \\
\hline $\begin{array}{l}\text { EE } 306(2) \\
\text { Advanced Programming }\end{array}$ & $\mathrm{X}$ & $\mathrm{X}$ & $\mathrm{X}$ & - & $\mathrm{X}$ & - & - & - \\
\hline $\begin{array}{l}\text { EE 310,310L }(3,1) \\
\text { Digital Logic \& Lab }\end{array}$ & $\mathrm{X}$ & $\mathrm{X}$ & $\mathrm{X}$ & $\mathrm{X}$ & - & $\mathrm{X}$ & - & - \\
\hline $\begin{array}{l}\text { EE } 320(3) \\
\text { Linear Systems }\end{array}$ & $X$ & $X$ & - & - & $X$ & $\mathrm{X}$ & $X$ & - \\
\hline $\begin{array}{l}\text { EE 321(3) } \\
\text { EM Theory }\end{array}$ & $\mathrm{X}$ & $X$ & - & - & $X$ & $\mathrm{X}$ & $\mathrm{X}$ & - \\
\hline $\begin{array}{l}\text { EE 330, 330L }(3,1) \\
\text { Electronics \& Lab }\end{array}$ & $\mathrm{X}$ & $X$ & $\mathrm{X}$ & $X$ & $\mathrm{X}$ & $X$ & $X$ & - \\
\hline $\begin{array}{l}\text { EE } 333(3)(\mathrm{TE}) \\
\text { Power Electronics }\end{array}$ & $X$ & $X$ & - & - & $X$ & - & $X$ & - \\
\hline $\begin{array}{l}\text { EE } 341 \text { (3) } \\
\text { Energy Conversion }\end{array}$ & $X$ & $\mathrm{X}$ & - & - & $X$ & - & $\mathrm{X}$ & - \\
\hline $\begin{array}{l}\text { EE } 342(3) \\
\text { Power Systems }\end{array}$ & $\mathrm{X}$ & $X$ & $\bar{X}$ & - & $X$ & - & $X$ & - \\
\hline $\begin{array}{l}\text { EE } 343 \text { (3) (TE) } \\
\text { Electric Power Distrib. }\end{array}$ & $\mathrm{X}$ & $X$ & - & - & $X$ & - & $X$ & - \\
\hline $\begin{array}{l}\text { EE } 350 \text { (3) } \\
\text { Communication Systems }\end{array}$ & $\mathrm{X}$ & $X$ & $\mathrm{X}$ & - & $X$ & - & $X$ & - \\
\hline $\begin{array}{l}\text { EE } 400,400 \mathrm{~L}(3,1) \\
\text { Control Systems I \& Lab }\end{array}$ & $X$ & $X$ & $X$ & $X$ & $X$ & $X$ & $X$ & - \\
\hline $\begin{array}{l}\text { EE } 401(3)(\mathrm{TE}) \\
\text { Control Systems II }\end{array}$ & $X$ & $\mathrm{X}$ & $\mathrm{X}$ & $X$ & - & $\mathrm{X}$ & $X$ & - \\
\hline $\begin{array}{l}\text { EE } 402 \text { (3) (TE) } \\
\text { Introduction to Robotics }\end{array}$ & $X$ & $X$ & $\bar{X}$ & $X$ & - & $X$ & $X$ & - \\
\hline $\begin{array}{l}\text { EE } 410(3)(\mathrm{TE}) \\
\text { Digital Signal Processing }\end{array}$ & $X$ & $X$ & $\mathrm{X}$ & $X$ & - & $X$ & $X$ & - \\
\hline $\begin{array}{l}\text { EE 410L (1) } \\
\text { Electrical Sys. Design lab }\end{array}$ & $X$ & $X$ & $\bar{X}$ & $X$ & $X$ & $\mathrm{X}$ & $X$ & $\mathrm{X}$ \\
\hline $\begin{array}{l}\text { EE } 415(3)(\mathrm{TE}) \\
\text { Intro. Digital VLSI }\end{array}$ & $X$ & $X$ & $\mathrm{X}$ & - & $X$ & - & - & - \\
\hline $\begin{array}{l}\text { EE } 430(3) \\
\text { Computer Structures }\end{array}$ & $X$ & $X$ & $\mathrm{X}$ & - & $X$ & - & - & - \\
\hline $\begin{array}{l}\text { EE } 431(3) \\
\text { Software Engineering }\end{array}$ & $\mathrm{X}$ & $\mathrm{X}$ & $\mathrm{X}$ & - & $\mathrm{X}$ & $X$ & - & - \\
\hline $\begin{array}{l}\text { EE } 432(3)(\mathrm{TE}) \\
\text { Computer Hardware }\end{array}$ & $\mathrm{X}$ & $\mathrm{X}$ & $\mathrm{X}$ & - & $X$ & - & - & - \\
\hline $\begin{array}{l}\text { EE } 450 \text { (1) } \\
\text { Capstone Design I }\end{array}$ & $X$ & $X$ & $\mathrm{X}$ & $X$ & $X$ & $\mathrm{X}$ & - & $X$ \\
\hline $\begin{array}{l}\text { EE } 451 \text { (1) } \\
\text { Capstone Design II }\end{array}$ & $X$ & $X$ & $\mathrm{X}$ & $X$ & $X$ & $X$ & - & $X$ \\
\hline $\begin{array}{l}\text { EE } 480 \text { (3) } \\
\text { Intro. Microprocessors }\end{array}$ & - & - & - & - & - & - & - & - \\
\hline $\begin{array}{l}\text { EE } 481 \text { (3) (TE) } \\
\text { Microproc. Applications }\end{array}$ & - & - & - & - & - & - & - & - \\
\hline $\begin{array}{l}\text { EE } 491(1) \\
\text { Senior Seminar }\end{array}$ & $X$ & $X$ & - & - & - & - & - & - \\
\hline
\end{tabular}


Table 3

COMPUTER FACILITIES IN THE COLLEGE OF ENGINEERING AND TECHNOLOGY (FOR GENERAL USE) AND IN THE ECE DEPARTMENT

\begin{tabular}{|c|c|c|c|c|c|c|c|c|c|}
\hline & \multicolumn{9}{|c|}{ CRITERIA FOR COMPUTER FACILITIES } \\
\hline $\begin{array}{l}\text { Computer- } \\
\text { Based } \\
\text { Lab Name \& } \\
\text { Room } \\
\text { Number }\end{array}$ & $\begin{array}{l}\text { MS-Dos, } \\
\text { Window-95 } \\
\text { Based } \\
\text { Machine } \\
\text { \& Number }\end{array}$ & $\begin{array}{l}\text { Unix Op. } \\
\text { Sys. } \\
\text { Based } \\
\text { Machine } \\
\& \\
\text { Number } \\
\end{array}$ & $\begin{array}{l}\text { Laser } \\
\text { and/or } \\
\text { InkJet } \\
\text { Printer } \\
\text { Facilities } \\
\text { \& Number } \\
\end{array}$ & $\begin{array}{l}\text { Structured } \\
\text { Language } \\
\text { Compilers } \\
\text { (QB,C, C++, } \\
\text { Fortran-77, } \\
\text { Pascal, etc) } \\
\end{array}$ & $\begin{array}{l}\text { Design, } \\
\text { Modeling, } \\
\text { Simulation \& } \\
\text { Analysis } \\
\text { Software } \\
\text { Packages } \\
\end{array}$ & $\begin{array}{l}\text { Data } \\
\text { Acquisition } \\
\text { Hardware } \\
\text { and } \\
\text { Software }\end{array}$ & $\begin{array}{l}\text { Technical } \\
\text { Help From } \\
\text { Competent } \\
\text { System } \\
\text { Support Staff }\end{array}$ & $\begin{array}{l}\text { Internet } \\
\text { Access } \\
\text { Facility }\end{array}$ & $\begin{array}{l}\text { Student } \\
\text { Accessibilit } \\
\text { y Between } \\
\text { 8am-12mid }\end{array}$ \\
\hline ENGR & \multicolumn{9}{|c|}{ AVAILABLE TO ALL ENGINEERING STUDENTS } \\
\hline $\begin{array}{l}\text { IBM Tutorial } \\
\text { Center } \\
\text { ET-122 } \\
\end{array}$ & 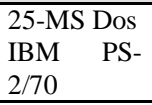 & No & $\begin{array}{l}\text { 7-Dot } \\
\text { Matrix }\end{array}$ & Q-Basic & Plato Tutorial & No & Yes & No & $\begin{array}{l}8: 00 \mathrm{am}- \\
12 \mathrm{mid}\end{array}$ \\
\hline $\begin{array}{l}\text { AT\&T } \\
\text { Freshmen } \\
\text { Lab } \\
\text { ET-116 } \\
\end{array}$ & $\begin{array}{l}25 \text {-Win95 } \\
\text { NRC-486 }\end{array}$ & No & $\begin{array}{l}\text { 1-Laser } \\
\text { 6-DeskJet }\end{array}$ & $\begin{array}{l}\text { Q-Basic, C, } \\
\text { C++, Fortran }\end{array}$ & $\begin{array}{l}\text { Matlab } \\
\text { PSpice }\end{array}$ & No & Yes & Yes & $\begin{array}{l}8: 00 \mathrm{am}- \\
12 \mathrm{mid}\end{array}$ \\
\hline $\begin{array}{l}\text { AT\&T CAD } \\
\text { Lab } \\
\text { ET-102 }\end{array}$ & $\begin{array}{l}\text { 34-Win } 3.1 \\
\text { IBM-Pentm }\end{array}$ & No & $\begin{array}{l}\text { 5-Plotter } \\
\text { 1-laser, 4- } \\
\text { DJ } \\
\end{array}$ & Q-Baisc & AutoCAD & No & Yes & Yes & \begin{tabular}{l}
\multicolumn{2}{l}{ Restricted } \\
Class Use \\
Only \\
\end{tabular} \\
\hline ECE & \multicolumn{9}{|c|}{ AVAILABLE TO ECE STUDENTS } \\
\hline $\begin{array}{l}\text { Neural Engr } \\
\text { Lab } \\
\text { ET-208 }\end{array}$ & $\begin{array}{l}\text { 7-Win95 } \\
\text { 2-Pent,5- } \\
486\end{array}$ & None & $\begin{array}{l}\text { 1-Laser } \\
\text { Printer }\end{array}$ & $\begin{array}{l}\mathrm{C}, \mathrm{C}++ \\
\text { Fortran }\end{array}$ & Matlab & None & No & No & $\begin{array}{l}\text { Research } \\
\text { Students } \\
\text { Only }\end{array}$ \\
\hline $\begin{array}{l}\text { Signal/Image } \\
\text { Proc. Lab } \\
\text { ET-205 }\end{array}$ & $\begin{array}{l}\text { 6-Win95 } \\
\text { Pentium }\end{array}$ & $\begin{array}{l}\text { 6-Unix } \\
\text { SUN }\end{array}$ & $\begin{array}{l}\text { 2-Laser } \\
\text { Printers }\end{array}$ & $\begin{array}{l}\text { C, C++, } \\
\text { Fortran }\end{array}$ & Matlab & $\begin{array}{l}\text { 2-National } \\
\text { Instr. A/D }\end{array}$ & No & Yes & $\begin{array}{l}\text { Research } \\
\text { Students } \\
\text { Only }\end{array}$ \\
\hline $\begin{array}{l}\text { Intelligent } \\
\text { Control Lab } \\
\text { ET-206 }\end{array}$ & $\begin{array}{l}\text { 5-Win95 } \\
\text { 2-Pent,2- } \\
486\end{array}$ & None & None & $\begin{array}{l}\mathrm{C}, \mathrm{C}++ \\
\text { Fortran }\end{array}$ & Matlab & None & No & No & $\begin{array}{l}\text { Research } \\
\text { Students } \\
\text { Only }\end{array}$ \\
\hline $\begin{array}{l}\text { Energy } \\
\text { Research } \\
\text { Lab } \\
\text { ET-212 } \\
\end{array}$ & $\begin{array}{l}\text { 3-Win95 } \\
\text { Pentium }\end{array}$ & $\begin{array}{l}\text { 2-Unix } \\
\text { SUN }\end{array}$ & $\begin{array}{l}\text { 2-Laser } \\
\text { Printers }\end{array}$ & $\mathrm{C}, \mathrm{C}++$ & Matlab & SUN A/D & No & Yes & $\begin{array}{l}\text { Research } \\
\text { Students } \\
\text { Only }\end{array}$ \\
\hline $\begin{array}{l}\text { Circuits Lab } \\
\text { ET-203 }\end{array}$ & $\begin{array}{l}\text { 8-MS Dos } \\
\text { IBM-386 }\end{array}$ & None & $\begin{array}{l}\text { 8-Dot } \\
\text { Matrix }\end{array}$ & Fortran, C & Matlab & $\begin{array}{l}\text { 8-Techmar } \\
\text { Micro. A/D }\end{array}$ & Yes & No & $\begin{array}{l}: \mathrm{am}- \\
4: 30 \mathrm{pm}\end{array}$ \\
\hline $\begin{array}{l}\text { Digital Lab } \\
\text { ET-206 }\end{array}$ & $\begin{array}{l}\text { 4-Win95 } \\
\text { 3-Pent,1- } \\
486\end{array}$ & None & None & None & Matlab & None & Yes & No & $\begin{array}{l}8: \mathrm{am}- \\
4: 30 \mathrm{pm}\end{array}$ \\
\hline $\begin{array}{l}\text { Control Lab } \\
\text { ET-202 }\end{array}$ & $\begin{array}{l}\text { 8-Win95 } \\
\text { All } 486\end{array}$ & None & $\begin{array}{l}\text { 1-Laser } \\
\text { Printers }\end{array}$ & $\begin{array}{l}\mathrm{C}, \mathrm{C}++ \\
\text { Fortran }\end{array}$ & Matlab & $\begin{array}{l}\text { 8-Feedback } \\
\text { A/D }\end{array}$ & Yes & No & $\begin{array}{l}\text { 8:am- } \\
4: 30 \mathrm{pm}\end{array}$ \\
\hline $\begin{array}{l}\text { Microproces } \\
\text { sor Lab } \\
\text { ET-201 }\end{array}$ & $\begin{array}{l}\text { 6-Win95 } \\
\text { Pentium }\end{array}$ & None & $\begin{array}{l}\text { 1-Laser } \\
\text { Printer }\end{array}$ & None & $\begin{array}{l}\text { LabVIEW, } \\
\text { Matlab }\end{array}$ & $\begin{array}{l}\text { 6-National } \\
\text { Instr. A/D }\end{array}$ & Yes & No & $\begin{array}{l}8: \mathrm{am}- \\
4: 30 \mathrm{pm}\end{array}$ \\
\hline
\end{tabular}




\begin{abstract}
APPENDIX
ABET Requirement of Computer Usage in Engineering (General) (Section iv.C.3.g.)

"Appropriate computer-based experience must be included in the program of each student. Students must demonstrate knowledge of the application and use of digital computation techniques for specific engineering problems. The program should include, for example, the use of computers for technical calculations, problem solving, data acquisition and processing, process control, computer-assisted design, computer graphics, and other functions and applications appropriate to the engineering discipline. Access to computational facilities must be sufficient to permit students and faculty to integrate computer work into course work whenever appropriate throughout the academic program."
\end{abstract}

ABET Criteria on Computer Usage for Specific Programs

The program criteria are specifically applicable to programs in specified disciplinary areas of engineering. These program criteria are also an integral part of the accreditation criteria.

Criteria on Computer Usage for ECE: (Amplifies criteria section IV.C.3.g, pp. 18)

Electrical Engineering: "Appropriate use of computers must be integrated with laboratory and course work. The program must include instruction in software design and must enable students to gain experience programming in a modern software development environment. Students also must be given access to several of the software packages available for engineering simulation, analysis, and design.”

Computer Engineering: "Computer use experiences must develop the student's proficiency in software design techniques and provide experience in several types of modern programming languages. The student must have experience using a range of design tools and be proficient with the advanced features of at least one major operating systems."

ABET Requirement of Computer Facilities in Engineering (General)(section IV.C.6.c)

"The computer facilities available to the engineering students and faculty must be adequate to encourage the use of computers as a part of the engineering educational experience. These facilities must be appropriate for engineering applications such as engineering computation, modeling and simulation, computer-assisted design, and laboratory applications. Students and faculty should have ready access to computational facilities. These facilities should have reasonable turnaround and response time and a competent support staff. The ultimate test of the computer facilities is the use made of them by the students and the faculty."

Computer Facilities as Stated in Program Criteria for Computer Engineering (Amplifies criteria section IV.C.6.c, pp. 18)

"The computer facilities must provide each student with ready access to a reasonable number and variety of hardware and software systems. These systems should have minimal downtime, reasonable response time, and should be administered by a competent systems support staff." 